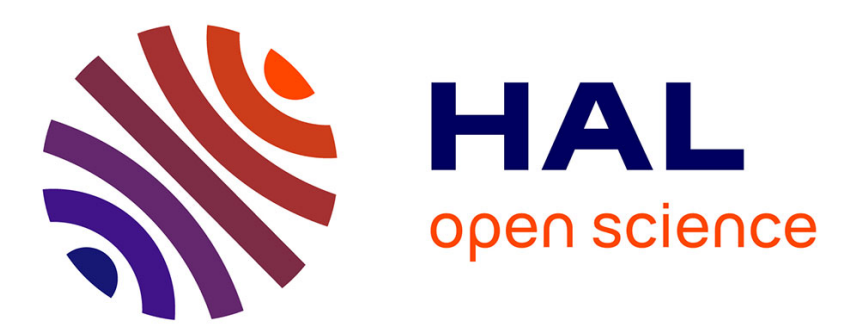

\title{
La cafétéria d'auges, méthode d'étude de l'appétibilité des fourrages
}

Michel Gillet, Claude Noël, Julien Jadas-Hecart, Rémy Traineau

\section{To cite this version:}

Michel Gillet, Claude Noël, Julien Jadas-Hecart, Rémy Traineau. La cafétéria d'auges, méthode d'étude de l'appétibilité des fourrages. Agronomie, 1983, 3 (8), pp.817-822. hal-00884575

\section{HAL Id: hal-00884575 \\ https://hal.science/hal-00884575}

Submitted on 1 Jan 1983

HAL is a multi-disciplinary open access archive for the deposit and dissemination of scientific research documents, whether they are published or not. The documents may come from teaching and research institutions in France or abroad, or from public or private research centers.
L'archive ouverte pluridisciplinaire HAL, est destinée au dépôt et à la diffusion de documents scientifiques de niveau recherche, publiés ou non, émanant des établissements d'enseignement et de recherche français ou étrangers, des laboratoires publics ou privés. 


\title{
La cafétéria d'auges, méthode d'étude de l'appétibilité des fourrages
}

\author{
Michel GILLET, Claude NOËL \& Julien JADAS-HECART \\ avec la collaboration technique de Rémy TRAINEAU \\ I.N.R.A., Station d'Amélioration des Plantes fourragères, F86600 Lusignan
} tester l'appétibilité dans les quelques heures qui suivent la coupe et opérer sous abri.

Mots clés additionnels : Graminées, sélection.

Palatability is the only intake character measurable on animals by the plant breeder. It is obtained by giving the animals free choice. Such a test is called cafeteria. It is usually carried out by grazing trials. There are some drawbacks to such grazing cafeteria particularly since it is not possible to measure yield and palatability in the same trial.

A trough cafeteria that avoids these drawbacks is presented here. Cheap and easy to utilize, it is reliable and very precise, provided a few precautions are observed, the two main ones being: testing palatability within a few hours after the cut, and working under a shelter.

Additional key words : Grasses, plant breeding.

\section{INTRODUCTION}

L'appétibilité est le nom donné à une caractéristique des fourrages : leur aptitude à exciter l'appétit de l'animal. On la mesure par des tests de consommation dans lesquels on donne à des animaux le choix entre plusieurs fourrages. De tels tests s'appellent des cafétérias.

Naturellement, si on donne le choix aux animaux, les résultats obtenus ne doivent pas être considérés en valeur absolue : c'est le classement entre les différents fourrages offerts que l'on cherche à connaître. De plus, rien ne prouve à priori qu'un fourrage préféré par des animaux qui ont le choix sera mangé en quantité abondante s'il est le seul aliment offert, ce qui est le cas habituel chez l'agriculteur. Néanmoins, les classements généralement obtenus pour l'appétibilité correspondent assez bien aux commentaires des agriculteurs qui ont des vaches laitières au pâturage ; de plus, l'appétibilité est liée à la digestibilité (GILLET \& NoËL, 1983). Ces considérations et quelques autres indices don- nent à penser que l'appétibilité a une bonne valeur de prédiction de la qualité des fourrages.

De toute façon, l'appétibilité est la seule caractéristique de consommation que le sélectionneur puisse mesurer avec des animaux sur quelques dizaines de génotypes à la fois, comme cela est nécessaire dans son travail. Pour la même raison, c'est actuellement la seule éventuellement utilisable pour les tests d'inscription à des catalogues.

Depuis longtemps, les sélectionneurs utilisent des cafétérias (PETERSEN et al., 1958 ; BUCKNER \& BURRUS, 1962 ; GILLET \& JADAS-HECART, 1965) : on enclôt un essai de variétés, on y lâche des animaux et, au bout de quelques heures, on note, à l'œil, la proportion d'herbe consommée sur chaque parcelle. Cette notation correspond bien à la réalité (PETERSEN, 1958; BUCKNER \& BURRUS, 1962 ; JADAS-HECART, 1982). Nous appellerons ce système : " cafétéria en pâture ». Il comporte certains inconvénients matériels qui nous ont amenés à rechercher une autre technique : la "cafétéria d'auges ». Après plusieurs années de tâtonnement, on peut dire que, moyennant quelques précautions essentielles, ce système est bien au point. 


\section{LA CAFIÉTÉRIA CLASSIQUE EN PÁTURE; SES INCONVÉNIENTS}

Les précautions à prendre avec la cafétéria en pâture sont les suivantes (la plupart, nous le verrons, sont valables pour toute cafétéria) :

- utiliser au moins 12 à 15 animaux d'âge et de poids variés, afin de s'affranchir des différences individuelles de préférences existant entre animaux,

- choisir des animaux calmes (mâles castrés par exemple) et, s'il s'agit de moutons, préférer une race pas trop grégaire. Les Texel conviennent parfaitement,

- s'assurer que, pendant 1 ou 2 jours au préalable, les bêtes aient à leur disposition un fourrage au moins aussi appétible que ceux que l'on veut tester. Si ces derniers sont tous nettement plus appétents que la nourriture de la veille, l'expérience montre en effet que les animaux ne choisissent pas. En fait, ce problème se pose rarement, car à la saison où on doit faire des tests d'appétibilité, les animaux pâturent généralement de l'herbe verte,

- inclure dans le parc une allée assez large autour de l'essai ( $2 \mathrm{~m}$ pour des moutons) afin d'y mettre un bac à eau et parce que les moutons ont tendance à rechercher l'abri des barrières contre le soleil ou le vent,

- avoir un essai ni trop petit ni trop grand en surface. S'il est trop petit, par rapport au nombre d'animaux, ceux-ci manquent de place ; s'il est trop grand, les bêtes risquent de ne pas en explorer la totalité pendant les quelques heures que dure le test. Nous utilisons des essais de 500 à $1000 \mathrm{~m}^{2}$, allées comprises, pour 12 à 15 moutons.

On surveille la progression de la pâture à partir de l'entrée des animaux sur l'essai et on effectue la notation lorsque celle-ci est la plus discriminante, en général quand à peu près la moitié de la masse d'herbe totale présente a été consommée (JADAS-HECART, 1982).

Ce système présente 2 inconvénients principaux.

- Il est peu précis, puisque le résultat est donné par la notation à l'oil, même si cette dernière est en bonne corrélation avec la réalité (JADAS-HECART, 1982).

- Surtout, il est impossible, sur un même essai, de mesurer le rendement et l'appétibilité en pâture. En effet, les parcelles utilisées en sélection sont trop petites pour qu'on puisse mesurer le rendement sur un simple prélèvement. Cela oblige donc à faire 2 essais au lieu d'un si on veut connaître les 2 caractéristiques sur un même ensemble de génotypes.

A priori, on pourrait croire à un $3^{e}$ inconvénient : l'effet du piétinement et des déjections pourrait modifier les classements. Mais l'expérience montre que cela joue peu, au moins lorsque la taille des parcelles n'est pas beaucoup plus grande que le corps d'un mouton: piétinement et déjections n'affectent pas alors forcément la même parcelle que celle qui est pâturée (JADAS-HECART, 1982).

C'est surtout pour pouvoir, sur un seul essai, mesurer rendement et appétibilité, que nous avons été amenés à mettre au point une cafétéria d'auges.

\section{PRINCIPE DE LA CAFÉTÉRIA D'AUGES}

Le principe de la cafétéria d'auges, très simple, est représenté figure $1:$ c'est un parc circulaire fait de barrières au travers desquelles les moutons peuvent facilement passer la tête. Les animaux sont introduits dans le parc juste après qu'on ait disposé les fourrages à tester dans de petites auges fixées autour du parc, à l'extérieur.

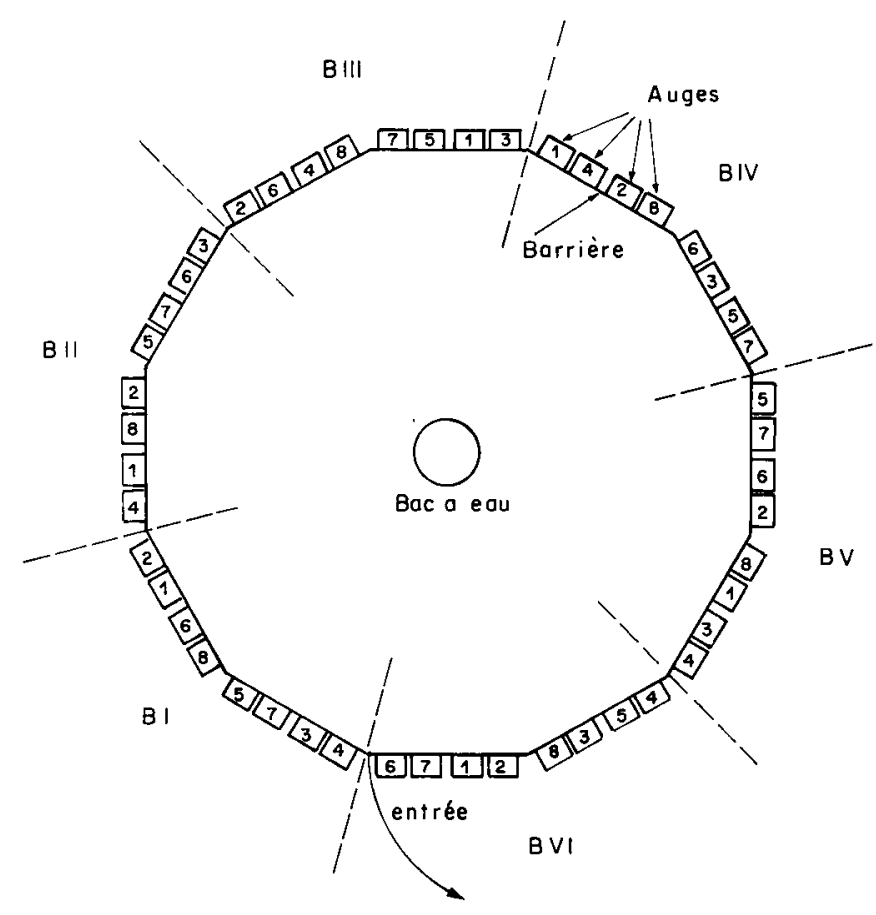

Figure 1

Plan de la cafétéria d'auges. BI, BII etc... les différentes répétitions. Lay-out of the trough cafeteria. BI, BII ... different replications.

Ces auges, en assez grand nombre (48 dans notre cas) permettent de diviser le périmètre en répétitions, à l'intérieur desquelles on tire au hasard l'affectation des auges aux variétés à tester (par exemple 8 variétés $\times 6$ répétitions). Il est d'ailleurs possible de faire correspondre chacune de ces répétitions avec une des répétitions de l'essai sur le terrain, si leur nombre est le même. L'analyse de variance est du type "essai bloc " classique.

\section{DESCRIPTION ET MODE D'EMPLOI DE LA CAFÉTÉRIA D'AUGES}

\section{A. Description}

La figure 2 montre comment sont faites les barrières et les auges. Les dimensions ont été établies par tâtonnements successifs. Pour les barrières, il faut que les moutons passent facilement la tête ; pour les auges, il s'agit d'éviter que les animaux laissent tomber par terre des brins de fourrage non consommés; il faut aussi que les auges ne soient pas trop profondes, afin que les animaux puissent voir d'assez loin s'il y a encore de l'herbe dedans.

Les barrières sont articulées entre elles grâce à des étriers métalliques dans lesquels passe un pieu métallique qu'on enfonce dans le sol. Une autre barre métallique sert de jambe de force. Elle est aplatie et percée aux 2 extrémités ; dans l'un des trous on passe le grand piquet ci-dessus avant de l'engager dans les étriers des barrières; dans l'autre trou, près du sol, on passe un petit piquet de fer, qu'on enfonce également.

Chaque barrière supporte 4 auges : 2 de chaque côté du montant central. Les auges sont accrochées sur les barrières par 2 fers courbes (fig. 2). Pour les vider, il suffit de les retirer en les soulevant.

Au centre du parc, on dispose le bac à eau.

Pour faire entrer ou sortir les animaux, aucune porte particulière n'est nécessaire : il suffit d'enlever le piquet qui joint 2 barrières et de faire pivoter l'une de celles-ci (fig. 1). 


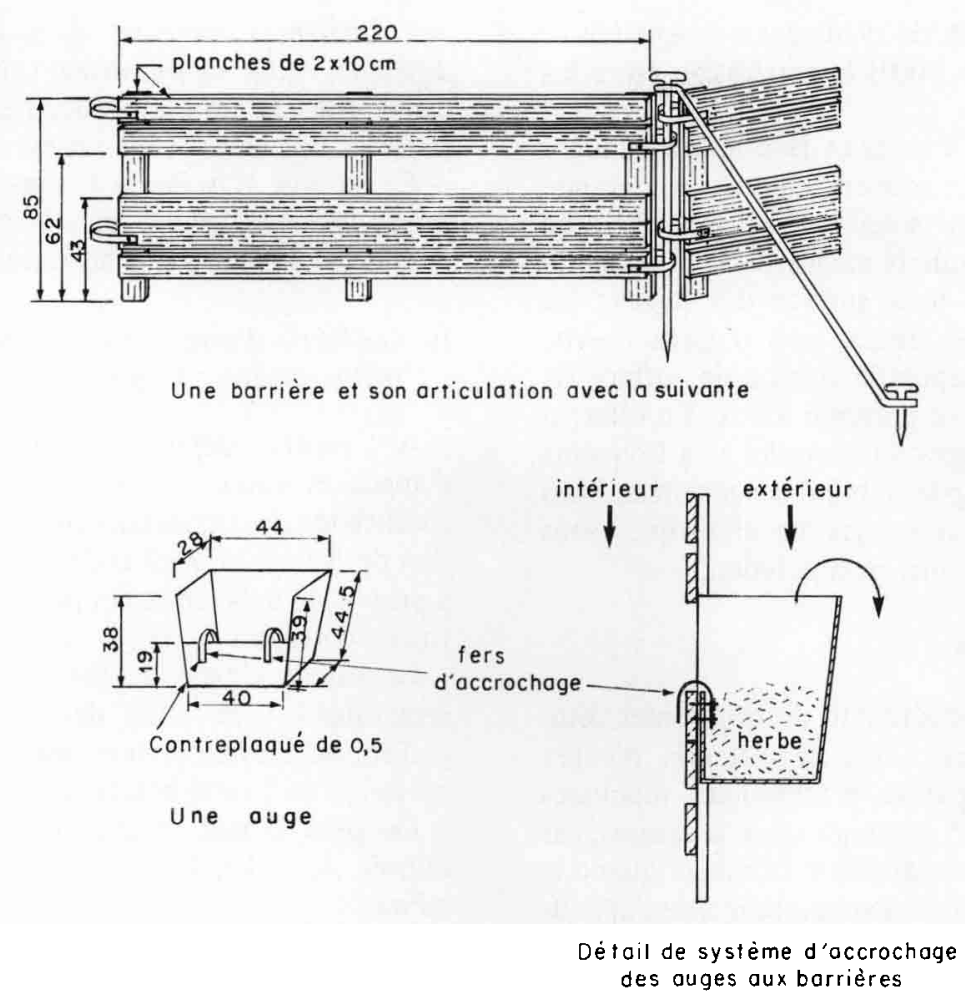

Figure 2

Description des barrières et des auges (les cotes sont exprimées en $\mathrm{cm}$ ).

Ce que nous avons dit plus haut à propos de la surface d'une cafétéria en pâture, s'applique encore ici (sauf l'allée extérieure, qui n'a pas de sens ici). Nous utilisons des parcs de 12 barrières (= 48 auges).

\section{B. Les animaux}

Le type d'animaux à utiliser, leur nombre, la nourriture à leur donner 1 ou 2 jours avant la mesure, sont les mêmes qu'avec la cafétéria en pâture. On retire les moutons quand les différences de consommation entre variétés semblent les plus nettes et on pèse les refus : à ce moment, les auges des variétés les plus appétibles sont presque vides, celles des variétés les moins appétibles ont tout juste été goûtées.

\section{Manipulation du fourrage}

On apporte dans chaque auge $1 \mathrm{~kg}$ d'herbe verte, de la variété affectée à cette auge par le tirage au sort. Il serait préférable d'apporter une quantité donnée de matière sèche, mais ce n'est pas possible, car on n'a pas le temps de déterminer le taux de matière sèche entre la coupe et la distribution.

On peut hacher l'herbe grossièrement (brins de $10-15 \mathrm{~cm}$ ) si elle est trop longue ; c'est inutile si elle est assez courte. De toute façon cela ne change rien aux classements. Il nous est arrivé de trouver une corrélation négative entre appétibilité en cafétéria de pâture et hauteur de l'herbe juste avant cette pâture: nous avons retrouvé cette corrélation avec l'appétibilité en cafétéria d'auges, même après avoir haché l'herbe.

L'analyse de variance se fait sur la matière verte consommée (1 kg apporté - refus). En effet, si 2 variétés ont des teneurs en matière sèche différentes, on apporte des quantités totales de matière sèche différentes. Si toutes deux sont également très bien consommées, les quantités consom-
Diagram of the barriers and troughs (in $\mathrm{cm}$ ).

mées, étant proches des quantités apportées, seront différentes si on les exprime en matière sèche. Il nous est arrivé de trouver des quantités de matière sèche consommées inférieures pour un ray-grass (riche en eau) que pour une fétuque visiblement moins appréciée (auges moins vides en fin d'essai, consommation moins rapide) mais plus riche en matière sèche ; le calcul sur le vert rétablissait l'ordre réel.

\section{Deux précautions fondamentales : distribuer l'herbe rapidement après la coupe et opérer sous abri.}

Si on distribue l'herbe le lendemain de la coupe, sans la mettre au réfrigérateur, on obtient des modifications d'appétibilité très importantes et impossibles à déterminer d'avance. Des tests d'appétibilité en cafétéria d'auges, effectués chaque jour sur le même ensemble de variétés, donnent des classements tout à fait incohérents d'un jour à l'autre si l'herbe a été coupée la veille de chaque test, alors que si l'appétibilité est mesurée le jour même des coupes, les classements restent constants.

Nous avons analysé ce phénomène en avril 1979. Le vendredi 6 avril, 14 variétés avaient été comparées en cafétéria de pâture sur un essai, pendant que leur rendement était mesuré par coupe d'un essai contigu et identique. L'herbe ainsi coupée a été conservée au réfrigérateur dans des sacs en jute (pour éviter la condensation) jusqu'au lundi 9 avril, date à laquelle cette herbe fut reprise pour faire une cafétéria d'auges. Entre temps, 3 variétés avaient chauffé (au réfrigérateur), une autre était franchement humide. La corrélation entre les 2 cafétérias était de 0,55 (S 5 p. 100) avec toutes les variétés, elle montait à 0,75 (S 1 p. 100) si on enlevait les 4 variétés mal conservées et mal consommées à l'auge. De plus, nous avons constaté, sur les variétés « bien conservées ", que les changements d'appétibilité entre les 2 dates (écarts à la régression) étaient liés à l'évolution des teneurs en matière sèche $(\mathrm{r}=0,65, \mathrm{~S} 5 \mathrm{p} .100)$. En corri- 
geant les données de la cafétéria d'auges en conséquence, on amenait à $r=0,91(\mathrm{~S} 1 \mathrm{p} .1000)$ la corrélation entre les 2 tests.

Il est également essentiel d'installer la cafétéria d'auges sous abri. Par temps calme et couvert, cela n'a pas grande importance, mais s'il y a du soleil ou un vent fort, on obtient, à l'extérieur, des résultats incohérents. Cela est dû à la dessication de l'herbe à la surface des auges: les animaux choisissent entre les auges, non d'après l'herbe qu'elles contiennent mais d'après la couche de surface de cette herbe, la première qui se présente à eux. En effet, si on brasse le fourrage des auges à intervalles très fréquents (1/4 h), on améliore beaucoup la stabilité des résultats. Mais cette manipulation est très contraignante alors que, sous abri, les résultats sont bons sans manipulation.

\section{E. Cas des fétuques élevées}

S'il s'agit de comparer l'appétibilité de différentes fétuques élevées, on aura intérêt, avec la cafétéria d'auges comme avec la cafétéria en pâture, à utiliser des repousses d'âge suffisant (au moins 4 à 5 semaines selon la saison), car les différences d'appétibilité apparaissent rarement quand la fétuque est trop jeune. D'ailleurs l'amélioration de l'appétibilité chez cette espèce n'a d'intérêt que pour améliorer sa souplesse d'exploitation, c'est-à-dire pour permettre le cas échéant des temps de repousse plus longs.

Chez les autres espèces de graminées, ce problème n'a pas été étudié.

\section{RÉSULTATS DE LA CAFÉTÉRIA D'AUGES}

\section{A. La cafétéria d'auges est très précise et fidèle}

Utilisée comme il est dit ci-dessus, la cafétéria d'auges est un instrument extrêmement précis. Sur 26 tests d'appćtibilité effectués avec cette technique de 1979 à 1982, les valeurs du $F$ de Fischer, pour 6 variétés et 8 répétitions, ont été comprises entre 20 et 339 ; le plus souvent elles étaient supérieures à 100 (la valeur limite du F au seuil de 1 p. 100 étant de 5,2). Dans ces tests, il y avait des fétuques élevées, un ou deux ray-grass et parfois un brome, mais la meillcure fétuque égalait souvent un ray-grass. Dans des tests de sélection ne comprenant que des fétuques (9 à 12 variétés $\times 4$ ou 5 répétitions), les $F$ sont généralement compris entre 5 et 20 (limite 1 p. $100=4,6$ ).

La cafétéria d'auges est également un test fidèle. En 1979, pendant 6 semaines, nous avons fait 4 tests d'appétibilité à l'auge par semaine sur les mêmes fourrages. Les interactions variétés $\times$ jours dans la même semaine sont significatives ( $\mathrm{F}$ compris entre 4 et 8 ) mais très significativement inférieures aux effets variétés (dont le $F$ est compris entre 60 et 260). Cela signifie que les irrégularités de classement d'un jour à l'autre sont mineures, comparées aux différences répétables entre variétés. De fait, on n'observe que de légères interversions entre variétés d'appétibilité presque égale, ou alors de simples variations dans les amplitudes des différences entre variétés, ce qui peut suffire à créer une interaction. Si donc ces interactions variétés $\times$ jours apparaissent significatives, quoiqu'insignifiantes, c'est à cause de la très grande précision de la méthode.

Naturellement, ces différences du jour au lendemain ne sont pas dues à des variations brusques d'appétibilité intrinsèque aux variétés, mais à de très légères interactions génotype $\times$ milieu. Cela signifie que, lorsqu'on fait un test d'appétibilité à l'auge, il ne faut pas accorder d'importance à des différences mineures dans les résultats, même si ces différences sont significatives, car elles sont susceptibles de disparaître du jour au lendemain. Seules, les différences relativement nettes sont vraiment d'origine variétale.

En résumé, la cafétéria d'auges est un test fidèle et sûr, et sa précision est si grande qu'elle met en évidence même des différences minimes qui ne caractérisent pas les variétés.

\section{B. Cafétéria d'auges et cafétéria en pâture expriment une même réalité : l'appétibilité}

A 4 reprises depuis 1979, nous avons comparé cafétéria d'auges et cafétéria en pâture. Le tableau 1 donne les coefficients de corrélation entre les 2 techniques (la corrélation du 5 avril 1979 est celle dont nous avons parlé ci-dessus à propos du délai entre coupe et distribution. C'est donc une valeur corrigée).

La valeur élevée de ces corrélations est d'autant plus remarquable que l'une des 2 variables, l'appétibilité en pâture, est inévitablement peu précise, puisqu'il s'agit de la moyenne de 5 ou 6 notations visuelles (une par répétition).

De plus, il faut préciser qu'à chaque fois, les moutons utilisés dans les 2 types de cafétérias n'étaient pas les mêmes.

Si les 2 techniques de mesure de l'appétibilité sont aussi ćtroitement corrélés, on peut en conclure que l'appétibilité est bien une caractéristique intrinsèque des fourrages et qu'on peut la mesurer à volonté avec l'une ou l'autre méthode.

\section{TABLEAU 1}

Coefficients de corrélation entre cafétéria d'auges et cafétéria de pâture.

Correlation coefficients between trough cafeteria and grazing cafeteria.

\begin{tabular}{rrr}
\hline Date & $\begin{array}{c}\text { Nombre } \\
\text { de variétés }\end{array}$ & $\begin{array}{c}\text { Coefficient } \\
\text { de corrélation }\end{array}$ \\
\hline $5 / 4 / 79$ & 14 & $0,91 \mathrm{SS}$ \\
$12 / 6 / 79$ & 9 & $0,77 \mathrm{SS}$ \\
$18 / 4 / 80$ & 5 & $0,99 \mathrm{SS}$ \\
$30 / 3 / 81$ & 12 & $0,84 \mathrm{SS}$ \\
\hline
\end{tabular}

\section{D'autres facteurs que l'appétibilité variétale influent sur les résultats d'une cafétéria. Il faut en tenir compte.}

L'appétibilité est influencée par les mêmes facteurs en cafétéria d'auges qu'en cafétéria de pâture: présence ou absence de tiges, attaques de maladies, hauteur d'herbe (même après hachage, voir IV), flexibilité du feuillage, aspect vert l'été (JADAS-HECART, 1982) et probablement d'autres facteurs, gustatifs ou autres. Il convient donc d'éviter de comparer des variétés ayant des proportions de tiges très différentes, ou des tiges plus ou moins avancées, car le résultat de la comparaison serait connu d'avance et tout à fait indépendant de l'appétibilité propre des fourrages. On évitera aussi d'introduire de l'herbe subissant une attaque importante de maladies; elle serait automatiquement refusée.

En ce qui concerne la hauteur d'herbe, on peut être amené à comparer, à stade égal, des variétés de port ou de rendement différents. Il faudra alors noter ou mesurer la 
hauteur de l'herbe sur chaque parcelle avant la pâture et établir la régression (ou dessiner le nuage de points) entre l'appétibilité et la hauteur d'herbe, afin de ne juger les variétés qu'à hauteur comparable.

A l'occasion de cafétérias d'auges où l'herbe était prélevée sur de très grandes parcelles (destinées à des études de quantités ingérées par moutons en cages, cf. GILLET \& NOËL, 1983) nous avons pu nous apercevoir que l'emplacement sur le terrain pouvait modifier l'appétibilité, sans doute par son influence sur la hauteur de l'herbe. Dans les tests habituels de sélection, cet inconvénient est pratiquement supprimé, puisqu'il y a des répétitions " terrain » et qu'en leur faisant correspondre les répétitions de la cafétéria, on juge l'effet variété par comparaison avec l'interaction variété $x$ terrain, contenue dans l'erreur résiduelle.

Rappelons enfin qu'il ne faut pas accorder d'importance à des différences mineures d'appétibilité, même significatives, car elles correspondent à de légères interactions génotype $\times$ milieu, susceptibles de disparaître du jour au lendemain : seules des différences relativement nettes sont réellement attribuables aux variétés et stables.

\section{CONCLUSIONS}

La cafétéria d'auges est un outil précis, fidèle et fiable, qui permet au sélectionneur de mesurer sur un seul et même essai le rendement et l'appétibilité d'une série de génotypes. Deux précautions essentielles sont à respecter ; faire le test d'appétibilité dans les quelques heures qui suivent la coupe et mettre le dispositif sous abri (ou brasser l'herbe tous les quarts d'heure).

Cet outil est très abordable pour la plupart des sélectionneurs. Sa construction est peu onéreuse, car l'appareillage est très simple. La seule chose qui puisse parfois poser un problème est de trouver un abri. Pour se procurer des moutons, il n'est pas nécessaire de posséder un troupeau : il suffit qu'il existe dans le voisinage des agriculteurs possédant des moutons, que l'on peut louer quelques jours par an. En effet, chaque essai n'est coupé que 4 à 6 fois par an et c'est à ces occasions qu'on mesure l'appétibilité.

\section{Reçu le 27 septembre 1982.} Accepté le 22 avril 1983.

\section{RÉFÉRENCES BIBLIOGRAPHIQUES}

Buckner R. C., Burrus P., 1962. Comparison of techniques for evaluating palatability differences among tall fescue strains. Crop Sci., 2 (1), 55-57.

Gillet M., Jadas-Hecart J., 1965. Leaf flexibility, a character for selection of tall fescue for palatability. Int. Grassland Cong., Sao Paulo, Section I, 595.

Gillet M., Noël C., 1983. Comment comparer l'ingestibilité en vert des variétés de graminées? Premiers résultats. Agronomie, 3 (7). Jadas-Hecart J., 1982. Etude en pâture de l'appétibilité de fétuques élevées (Festuca arundinacea Schreb.) à l'aide de moutons. Agronomie, 2 (5), 487-492.

Petersen R. C., Weswig P. H., Cowan J. R., 1958. Measuring palatability differences in tall fescue by grazing sheep. Agron. $J ., 50$ (3), 117-119. 